\title{
La défense des forêts contre les insectes. Approches actuelles et perspectives
}

\author{
J. LEVIEUX \\ INRA, Station de Zoologie forestière \\ Centre de Recherches d'Orléans, Ardon, F 45160 Olivet
}

\section{Résumé}

Les attaques d'insectes subies par certaines de nos forêts depuis une décennie conduisent à s'interroger sur la validité des méthodes actuelles de protection contre les ravageurs et sur les perspectives offertes par diverses recherches en cours.

Au plan préventif, quelques modifications des méthodes sylvicoles pourraient apporter des résultats plus rapides que ceux à attendre de la sélection génétique de variétés résistantes. Les études dans ce dernier domaine sont encore trop peu avancées pour être susceptibles, sauf exceptions notables, d'une généralisation rapide.

Les méthodes curatives sont appliquées avec des succès variés. L'utilisation d'insecticides choisis, très positive à courte échéance contre certains phyllophages, reste décevante vis-à-vis des xylophages. La mise en ceuvre raisonnée de pièges à phéromones aux densités de populations faibles ou moyennes peut aider à contrôler la densité de certains ravageurs. On tente aussi de développer plusieurs procédés modifiant la physiologie de l'insecte cible en perturbant l'une ou l'autre des étapes de son développement (analogues d'hormones juvéniles, dichromères, inactivateur de cette hormone, benzoylurées...).

Quelques méthodes de lutte biologique peuvent être employées avec un certain succès sans toutefois obtenir de résultats spectaculaires. L'utilisation des Coléoptères Rhizophagus contre le Scolyte Dendroctonus paraît cependant prometteuse. Divers agents pathogènes ont été essayés (microsporidies, baculovirus...). B. thuringiensis, bien que la rémanence des préparations soit souvent brève, est efficace contre plusieurs Lépidoptères. Les travaux sur un éventuel contrôle génétique d'insectes n'ont pas dépassé le stade des études préliminaires.

Par suite, il paraît plus que jamais indispensable de concevoir et d'appliquer à la forêt des approches de lutte intégrée unissant, outre les efforts du sylviculteur, ceux des généticiens et chercheurs fondamentalistes pour créer une synergie entre procédés existants.

Mots clés : Protection, forêt, insecte, revue, méthode.

\section{Introduction}

C'est ce que nous ne faisons pas plutôt que ce que nous faisons qui rend les traitements appliqués aux arbres inefficaces. En effet, nous ne modifions pas fondamentalement les conditions qui sont les causes de l'apparition des problèmes.

Coulson R.N. \& StaRK R.W., 1982.

On sait l'ampleur des problèmes posés à nos forêts par les insectes et par les champignons qu'ils véhiculent. Cette situation se répète épisodiquement dans l'histoire des massifs ; elle conduit à s'interroger sur l'orientation des actions à développer et le 
type des procédés à mettre en œuvre pour, sinon supprimer ces phénomènes ce qui paraît illusoire, tenter à tout le moins d'en circonscrire les dégâts. On trouvera donc dans les pages qui suivent une description commentée de l'état actuel du développement de quelques méthodes et divers éléments de réflexion qu'elles inspirent.

Il est commun d'user dans le langage courant des termes «aménagement»ou «contrôle » au sujet des ravageurs sans toujours préciser de façon explicite dans quels cadres de gestion ils s'insèrent. Le gestionnaire se doit de considérer chaque fois que possible, outre les aspects de protection, ceux de conservation du milieu naturel. Cependant, si les aspects de protection sont à prendre en compte dès l'installation d'un dispositif, ils ne sauraient être les seuls. Il se peut même que l'éventuel aménagement d'une surface forestière vis-à-vis d'une espèce nuisible puisse aller à l'encontre du but recherché. Toute espèce nuisible ne constituant que l'une des composantes fonctionnelles d'un écosystème, son élimination laisse le champ libre à d'autres phytophages présents dans ce système. On saisit donc toute la complexité du sujet.

Compte tenu de l'évolution actuelle des concepts de protection phytosanitaire des forêts, il nous a paru intéressant de résumer les acquis des expériences conduites par divers chercheurs en plusieurs points du globe pour dégager quelques principes d'action dont on souhaite qu'ils soient intégrés aux démarches entreprises pour sauver nos forêts.

\section{Constat}

Un certain nombre de difficultés s'ajoutent pour rendre délicate toute approche de lutte contre les ravageurs forestiers et empêcher la généralisation de méthodes existantes ou à l'essai.

Compte tenu de l'ancienneté et de la densité de l'implantation humaine en Europe, il n'y existe plus depuis longtemps de forêts dites " naturelles », encore moins de forêts considérées comme «vierges" (BECKER \& LE TACON, 1985). Pour bien des auteurs, l'artificialité des forêts actuelles engendre un degré de sensibilité aux ravageurs plus élevé que celui des sylves naturelles. Ce point mérite d'être commenté.

Le degré de résistance d'une forêt dépendant d'abord de sa diversité floristique c'est-à-dire de son degré de mixité, les forêts naturelles, souvent à large spectre floristique, seraient moins attaquées. C'est quelquefois le cas. Cependant, la sapinière canadienne, naturelle dans sa majeure partie, composée d'une cinquantaine d'espèces de feuillus et de résineux en mélange introduisant une certaine diversité, subit l'impact de divers ravageurs dont la Tordeuse des bourgeons de l'Epinette (Choristoneura fumiferana Clem.). Notons cependant que les espèces d'arbres sensibles constituent souvent 80 p. 100 des peuplements. L'importance des attaques est telle que les services forestiers canadiens ont délibérément brûlé en une seule fois 800 hectares de forêt dans l'Ontario en août 1985 pour détruire un foyer d'infestation. De même l'étendue des zones attaquées par les Scolytes dans les forêts naturelles peu ou pas exploitées du continent nord américain ne cesse de s'agrandir. Ainsi, ce seul critère parait-il insuffisant pour assurer l'immunité.

Les écosystèmes forestiers artificiels, souvent monospécifiques, présentent la plupart du temps une certaine fragilité (SchwerdtFiger, 1954 ; Voute, 1964). Ceci reste 
valable que l'arbre soit d'origine indigène ou exotique. Par exemple, l'Epicéa commun, planté sur sols acides à faibles réserves en eau, dépérit en année sèche. Ceci favorise l'explosion des populations d'Ips typographus qui, à partir des placettes où les résineux sont mal implantés, gagnent celles où il est mieux adapté (Becker \& LE Tacon, 1985). Les Pins sylvestres installés sur les parcelles les plus hydromorphes de la Forêt d'Orléans ont subi durant une décennie l'impact de divers ravageurs (Diprion du Pin, plusieurs Scolytes...) ayant causé d'importants dégâts. Les conditions de milieu semblent avoir joué ici un rôle déterminant.

A l'inverse, la monospécificité ne conduit pas obligatoirement à des catastrophes, du moins à l'échelle d'observations qui est la nôtre. Le Massif des Landes n'a pas subi depuis 40 ans de dégâts majeurs bien que la plupart des ravageurs cités des autres forêts françaises y soient présents en permanence (Scolytes, Matsucoccus...). La provenance des arbres, pas plus que l'humidité relative ambiante nécessaire à une bonne croissance du Pin maritime, n'expliquent la relative inocuité dont semble bénéficier l'essence dans cette zone géographique. Les récents déboires subis dans une partie du massif sont des résultantes directes du gel de l'hiver 1985.

Un autre facteur nécessite examen. II consiste dans l'introduction sous nos latitudes d'espèces exotiques. Certains essais récents paraissent être dans une première phase des succès économiques sans contreparties phytosanitaires majeures. C'est le cas du Douglas. Notons cependant l'importance du facteur temps dans ce domaine. Comment évoluera le pouvoir adaptatif du puceron Gilletteella cooleyi dans les décennies à venir ? Cet aphidien semble s'adapter aux Epicéas de Sitka comme aux jeunes Douglas (Voute, 1964). Plus généralement, on peut redouter le résultat de la pression de sélection considérable qu'exerce sur l'entomofaune phytophage l'existence de vastes massifs monospécifiques d'essences exotiques auxquelles les insectes locaux sont étrangers au départ. Il peut y avoir à la longue apparition de populations locales de ravageurs adaptés à ces essences. Il en est de même sous d'autres latitudes. Ainsi, Madagascar, île dépourvue de Conifères indigènes, abrite un Lépidoptère Lymantriidae inféodé aux Angiospermes qui s'adapte progressivement aux Pins, causant des dommages considérables aux boisements artificiels ( $P$. du Merle, comm. pers.).

Bien d'autres points seraient à considérer qu'il serait trop long d'examiner ici. Des connaissances plus approfondies sur la constitution du patrimoine génétique des hôtes et de leurs ravageurs, sur l'influence du milieu, sur la sensibilité physiologique des tiges aux attaques dans des conditions climatiques et édaphiques connues, sur la nature exacte et le mode de fonctionnement des mécanismes de défense des arbres seront indispensables pour élaborer des méthodes de protection préventives et curatives adéquates comme pour expliquer le passage d'un niveau de population d'une phase d'endémie à une période d'épidémie.

Deux catégories de facteurs interviennent conjointement ou successivement pour causer des dégâts :

- des facteurs catastrophiques (tempêtes, sécheresse, froid, guerre...) dont la durée d'action est plus ou moins brève et qui nécessitent de la part du gestionnaire des actions importantes et brutales;

- des facteurs diffus jouant à plus ou moins longue échéance. Leur influence est permanente sinon durable. Il convient alors de leur opposer des moyens humains appliqués au long des années.

Selon l'essence, le milieu, l'époque, et sans oublier le rôle fondamental de la surveillance puis de la prognose, le gestionnaire doit choisir entre des moyens curatifs 
souvent mécaniques ou diverses autres méthodes chimiques ou biologiques. Par suite le texte qui suit voudrait rappeler au forestier les difficultés rencontrées, exposer plusieurs moyens de lutte existants ou à l'essai et présenter quelques pistes de recherches dont on souhaite qu'elles débouchent sur des méthodes de protection applicables.

\section{Les moyens}

Les moyens préventifs relèvent plutôt de la sélection et de la sylviculture, les curatifs sont d'abord basés sur une lutte mécanique, chimique ou biologique. Depuis une décennie, on tend à associer cet ensemble pour mettre en œuvre une lutte intégrée contre les ravageurs.

\subsection{Procédés préventifs}

\subsection{Influence du milieu et des procédés culturaux}

On sait depuis longtemps l'avantage procuré par une bonne adéquation de l'essence au site d'installation (Le CAM, 1983; BeCKER \& LÉvy, 1983). Outre un avantage productif, ceci favorise la croissance d'arbres plus vigoureux dont la sensibilité à certaines attaques pourrait être diminuée. C'est parfois le cas face aux Scolytes. On ne saurait malheureusement généraliser cette assertion à la plupart des phyllophages dont on ignore le plus souvent les modalités du choix lors de leur installation sur les tiges. Quoi qu'il en soit, le milieu exerce toujours une influence déterminante. En forêt de Tronçais, le chêne pédonculé dépérit alors que le rouvre reste sain. Plusieurs facteurs interviennent dans la définition des caractéristiques du milieu propres à fournir des arbres résistants à divers ravageurs (voir Houston, 1983 ; WrTter et al., 1983 sur la résistance à Lymantria ou à Choristoneura). Les modifications de l'éclairement peuvent parfois conduire au même résultat: une augmentation notable de l'éclairement au sol, en jouant sur l'espacement des plants, diminue de façon significative les attaques de Pissodes strobi (STiell \& Berry, 1985). Dans le même ordre d'idées, les pullulations de L. dispar semblent liées aux climats secs et lumineux des formations ouvertes (Fraval et al., 1980). Dans bien des cas, cependant, i ne semble pas exister de relations évidentes entre la densité de la végétation et l'intensité des attaques. Il en est ainsi dans l'étude réalisée par Milıer \& Stephen (1983) sur le niveau d'attaque de Rhyacionia frustrana (Const.) dans des plantations de Pinus taeda. On sait enfin le rôle des pollutions déclenchant des pullulations de la Nonne en Europe de l'Est. Des techniques culturales appropriées réduisent l'impact des ravageurs. Les populations du Cerambycidae Enapholades, foreur du chêne rouge d'Amérique, sont diminuées de 65 p. 100 par un léger abattage sélectif (Donley, 1983). L'aménagement raisonné des parcelles de Pinus contorta peut diminuer l'importance des attaques de Dentroctonus (Coulson \& STARK, 1982). L'éclaircie des $P$. contorta augmente la vigueur des arbres et leur résistance à Dendroctonus ponderosae (Mrtchell et al., 1983). De même, une sylviculture appropriée réduirait les pertes dues à D. frontalis (Belanger, 1980). Plusieurs approches sylvicoles sont d'ailleurs proposées contre les scolytes (Branham \& THATCHER, 1985). A l'inverse, l'obtention d'une futaie pure de Hêtre favorise la propagation 
de la cochenille Cryptococcus (Perrin, 1979). Turchinskaya (1983) décrit une série de pratiques sylvicoles évitant les invasions d'Hylobius abietis. Le contrôle de la présence de «mauvaises herbes » joue aussi un rôle. Pour se limiter à un seul exemple, la présence de Lupin modifierait la densité de Rhyacionia buoliana dans les jeunes plantations de Pin sylvestre (Rodziewicz \& Kolk, 1980 ; Kelleher \& Hume, op. cit., $2^{\circ}$ partie).

L'inadéquation de l'essence au sol favorise aussi l'action de certains ravageurs. Les Pins sylvestres plantés sur les calcaires de Champagne ont subi les attaques du Lépidoptère Lasiocampidae Dendrolimus pini, ravageur fréquent du Pin sylvestre dans l'Est de l'Europe (JACQuiot, 1983). Dans la région d'Uzès, lors d'une pullulation de Lymantria dispar, seuls les plants situés sur calcaire néocomien ont été envahis, ceux poussant sur le loess restant indemnes. La sensibilité des plants aux attaques selon le type de sol serait liée au pouvoir de rétention en eau de ce dernier (CoInTAt, 1948). Plus généralement, on sait le rôle joué par les organismes édaphiques sur le fonctionnement des sols et le retentissement de leur action sur l'état des flores qu'ils abritent (Berthelin \& Toutain, 1979 ; Ponge, 1983, 1986).

On ne saurait trop rappeler ici les avantages présentés par l'introduction d'une certaine mixité dans les peuplements. Celle-ci peut être basée sur un mélange d'espèces ou de provenances de la même espèce, par exemple un hêtre maintenu en sous-étage par recépage pour 10 Pins sylvestres comme à Bitche. Si elle pose dans la pratique un certain nombre de problèmes de gestion conduisant le forestier à planter les différentes espèces en lignes ou en bouquets d'une vingtaine d'hectares par exemple, elle contribue à la diversification de la flore et par suite de la faune comme au maintien des équilibres humiques par un choix judicieux des essences. Ainsi, les forestiers polonais tentent de conserver à l'intérieur de leurs massifs monospécifiques, par exemple autour d'un point d'eau, des parcelles unitaires de 0,3 à 0,5 ha pour 10 ha de massifs. Celles-ci conservent une partie de la flore originelle comme le Tilleul et sont enrichies en essences mellifères introduites (Robiniers, Prunus serotina...). Elles servent ainsi de refuge à la faune locale d'insectes, de rongeurs ou d'oiseaux et facilitent le développement et l'entretien des cortèges de prédateurs et de parasites des ravageurs communs (méthode des «Places complexes», Birot et al., rapport de mission INRA en Pologne, 1985). Rappelons aussi pour mémoire, l'effet positif du Charme dans le maintien des équilibres humiques, maintien qui subsiste longtemps après l'élimination de ce feuillu (Ponge, rapp. Piren, 1985).

Le rôle du sylviculteur ne saurait se limiter à implanter la bonne provenance dans un sol qui lui convient. Il lui appartient aussi de produire du bois. Pour ce faire, l'une des tendances actuelles consiste, comme en agronomie, à engraisser le sol pour intensifier la production. Quelles sont les retombées pratiques d'une telle intensification?

Chez les Pins Laricio de Corse plantés dans le Lot avec engraissement (fumure de fond $\mathrm{P}, \mathrm{K}, \mathrm{Mg}$ avant la plantation, fumure $\mathrm{N}, \mathrm{P}, \mathrm{K}$ après), on constate des cas de fourchaisons. Celles-ci peuvent être dues à quatre causes : la constitution génétique, le polycyclisme, l'impact de la Tordeuse des pousses (Rhyacionia buoliana), divers accidents dus aux oiseaux. Les trois premières causes peuvent être reliées au patrimoine héréditaire, 71 p. 100 des arbres mesurés possèdent ce défaut qui est 2 à 3 fois plus fréquent que dans les boisements traditionnels. Cette augmentation de fréquence découle probablement de l'intensification culturale (Roman AMat, comm. pers.). De même, le pourcentage de fourchaisons chez $P$. pinaster paraît être fortement corrélé 
avec l'augmentation des doses de $\mathrm{P}_{2} \mathrm{O}_{5}$ : le pourcentage d'arbres attaqués par Dioryctria augmente dans les mêmes proportions (AlazARD. 1980).

\subsection{Procédés dus à l'amélioration génétique}

L'approche génétique peut porter sur le ravageur (voir plus loin) mais aussi sur l'hôte par sélection de provenance. Il existe chez les plantes divers types de résistance à contrôle mono ou polygéniques. Les résistances monogéniques, parfois qualifiées de "verticales " (VAN DER PLANK, 1975) sont absolues mais aisées à contourner. Les oligoou polygéniques, quelquefois appelées «horizontales» ou uniformes, sont gouvernées par des gènes mineurs. Leurs actions combinées confirment une résistance partielle (Clerjeau et al., 1979, 1981). Si les pathologistes forestiers obtiennent actuellement quelques succès contre des champignons phytopathogènes (PINON, 1984, 1986), bien des points restent à élucider en ce qui concerne les résistances éventuelles des arbres vis-àvis des insectes, les approches actuelles des entomologistes étant souvent descriptives. Il se pose de plus la question de la stabilité temporelle de la résistance acquise compte tenu de la durée de vie des ligneux forestiers.

Il existe une variété de pommiers résistante au Puceron lanigère. Dans ce cas, il s'agit de porte-greffes. La création de variétés résistantes est plus récente : on cultive depuis 40 ans un framboisier résistant aux Pucerons ou un pommier résistant à la tavelure (KEEP, 1976 ; LESPINASSE et al., 1976). On attend des résultats analogues pour le pêcher (Massonié, 1977). Cette résistance peut cependant être surmontée ; ainsi, il s'est écoulé plus d'un siècle avant que les premiers biotypes de Pucerons capables de tourner la résistance conférée par le gène $\mathrm{E}_{\mathrm{r}}$ chez la variété Northern Spy du pommier soient signalés (Gilomme et al., 1968).

Dans le domaine forestier, il ne semble pas illusoire de penser à sélectionner de provenances moins sensibles (Levifux, 1986). Par exemple, on soupçonne chez le Pin maritime lexistence de clones riches en limonène dont la résistance à Dioryctria serait plus marquée (Bernard Dagan \& Baradat, 19?7). On sait aussi l'importance de la provenance pour diminuer la sensibilité des Pinus aux attaques de la Cochenille Matsucoccus ( $P$. halepensis à $M$. josephi: MENDEL, $1984 ; P$. pinaster à $M$. feytaudi : Bernard Dagan \& Baradat, 1977 ; Schvester \& Ughetto, 1986). Chez les Conifères, la résistance aux Scolytes comme à leurs champignons associés serait due à l'existence d'un système chimique défensif complexe mettant en jeu plusieurs molécules (terpènes, phénols antifongiques, acides résiniques, leucoanthocyanidines...) (CATEs \& Alexander, 1982). Les performances liées à la résistance résulteraient pour un arbre donné d'une interaction entre le génotype de l'arbre et son environnement.

Plus généralement, il existe un certain nombre de travaux décrivant des phénomènes de résistance d'arbres forestiers aux attaques d'insectes (RoTH, 1970 ; TODA, 1974 ; Hanover, 1976 et 1980 ; Russell, 1978). Limitons-nous à quelques exemples.

On doit à STEINER (1974) puis à Wright et al. (1976) une étude comparée de la résistance relative en Amérique du Nord de 108 variétés de Pin sylvestre aux attaques d'Hylobius radicis. Il existe des différences importantes de sensibilité selon les variétés : ainsi, dans une provenance de la variété laponica, 14 p. 100 des arbres sont tués par les attaques. Pour une provenance sibérienne de la variété uralensis, cette valeur monte à 50 p. 100. Les auteurs n'ont cependant pu relier aucune caractéristique morphologique ou biochimique de l'hôte au degré de résistance et ils estiment, compte tenu de lampleur de la variation naturelle que les améliorateurs devraient pouvoir sélectionner des variétés encore plus résistantes. Il reste à s'interroger sur la validité de comparai- 
sons de sensibilité faite en plantations comparatives, comparaisons peu extrapolables lorsque l'on passe aux plantations habituelles. Dans le Michigan, 2,2 p. 100 des variétés scandinaves et sibériennes sont infestées par Neodiprion sertifer contre 21 p. 100 pour les arbres issus d'Europe Centrale (WRIGHT et al., 1976). On trouve des valeurs du même ordre de grandeur vis-à-vis des attaques de Dioryctria zimmermani. Les arbres résistants possèdent souvent des teneurs exceptionnellement basses en certains terpènes comme le 3 carène ou le terpinolène sans que l'on puisse établir pour l'instant une relation de cause à effet. Comme dans les autres essences, il n'existe au total aucune variété de Pin sylvestre complètement résistante aux attaques d'insectes; on constate cependant des différences intervariétales frappantes vis-à-vis de chaque ravageur. Malheureusement, la résistance individuelle de l'arbre à chaque ravageur semble être indépendamment héritable et une variété résistante dans un cas donné l'est beaucoup moins vis-à-vis d'un autre agresseur. L'améliorateur doit donc sélectionner, dans un premier temps, les plants en fonction des dangers potentiels existants dans la zone prévue de plantations. A très longue échéance, l'espoir serait permis de sélectionner des variétés peu sensibles aux principaux insectes d'une zone biogéographique donnée (Russell, 1978).

\subsection{Procédés curatifs}

\subsection{Procédés de lutte physique}

Des procédés physiques ou mécaniques fort variés ont été essayés pour protéger les jeunes plants ou les arbres. Citons quelques exemples. Des colliers en résille entourent le collet de jeunes pins ou Epicéas pour limiter les dégâts dus aux Pissodes (Lindstrom et al., 1982). Ce procédé peut évidemment être utilisé préventivement. Des nébulisations d'eau froide modifieraient les dates d'éclosion des bourgeons de Pseudotsuga menziesii pour diminuer les taux d'attaque d'un Diptère Ceccidomyidae Contarina oregonensis Foote (MiLler, 1983). L'effet obtenu serait analogue à celui d'une pulvérisation de diméthoate. Pour contrôler Dendroctonus ponderosae, on a pensé à écorcer les Pins poussant autour des arbres infestés. Pour ce faire, un ébrancheur écorceur a été conçu puis mis en œuvre. Le rapport coût-efficacité serait prometteur (Moulson, 1985). Divers procédés techniques utilisés pour lutter contre Dendroctonus sont exposés dans Klein (1978; voir aussi Safranyik \& Linton, 1982). En désespoir de cause et devant l'ampleur des dégâts causés par ces Coléoptères, on a même pensé à les électrocuter sur les arbres debout ou à enrouler des cordons explosifs autour des troncs !... Pour originaux qu'ils paraissent, l'utilisation de ces procédés n'a pas encore été généralisée.

\subsection{Procédés mettant en jeu des molécules}

- Insecticides :

Après l'utilisation 400 ans avant Jésus-Christ du Pyrèthre en Perse (Moore in QuRAISHI, 1977), l'application vers 1734 de la nicotine en solution aqueuse contre un charançon puis en 1848 de la roténone contre une chenille phyllophage préfigure l'approche actuelle. De nos jours, l'éventail des molécules disponibles s'est grandement élargi, mais l'utilisation raisonnée de ces produits reste indispensable.

Depuis 30 ans, l'industrie chimique a largement complété l'éventail des molécules naturelles. Les grandes familles moléculaires à l'origine des produits utilisés possèdent des spectres et des modes d'action assez différents. Les organochlorés sont peu 
spécifiques. D'autres insecticides sont systémiques ou présentent une faible toxicité visà-vis des Mammifères (Malathion, Abate). Ils agissent par blocage d'une cholinesterase jouant un rôle fondamental dans l'hydrolyse de l'acétylcholine (NArahashi, 1965). Les carbamates, inhibiteurs de l'acétylcholine ont un spectre plus étroit. Ils sont rapidement métabolisés et toxiques pour les Mammifères (Baygon, Aldicarb, Carbofuren). Le D.D.T. est trop connu pour que l'on s'y attarde. En forêt, il s'est révélé très efficace contre Lymantria dispar. Il en existe maintenant des analogues biodégradables. Les autres organochlorés (Aldrin, Dieldrin, Endosulfan, Mirex) suivent des voies métaboliques compliquées. Certains sont très stables. Le Lindane, rapidement métabolisé, est dangereux pour les Mammifères surtout par voie respiratoire.

La lutte par voie chimique rencontre dans le domaine forestier des résultats pour le moins variables. En ce qui concerne les Scolytes, un contrôle effectif des populations d'Ips subelongatus aurait été obtenu en Chine par l'usage de Lindane, de Dichlorvos voire même d'huile de diesel ! (Yu et al., 1984). L'emploi d'insecticides pourrait avoir un effet préventif en limitant l'impact des attaques de Scolytes sur les arbres traités (GiBSON \& BENNETT, 1985). A l'inverse, on n'a pas pu mesurer l'éventuelle influence d'un traitement à la Deltaméthrine effectué en Forêt d'Orléans contre les populations d'Hylésine du Pin (Sauvard, comm. pers.). Il convient de rester prudent en ce domaine quant à l'ampleur des résultats généralement obtenus. La majorité des expérimentateurs s'accorde cependant pour reconnaître l'inefficacité du contrôle chimique contre les Scolytes en forêt (Coulson \& STARCK, 1982).

Les résultats obtenus semblent plus positifs en ce qui regarde les phyllophages où il existe une importante bibliographie. Ainsi, un certain contrôle des populations de Lymantria dispar est obtenu par pulvérisation aérienne de Carbaryl (Robredo \& SANChez, 1983 ; CAmERon et al., 1983).

L'un des problèmes essentiels réside dans la dégradation des molécules étrangères au fonctionnement normal de l'écosystème. Qu'on se rappelle seulement l'impact sur les populations de saumons canadiens des applications répétées de D.D.T. contre le Tordeuse du bourgeon de l'Epinette. Ceci explique pour partie le remplacement de molécules efficaces par d'autres composés plus onéreux, comme le Fénithrition ou le Matacil, se dégradant plus vite dans le milieu (PrebBle, 1976).

Avant toute intervention dans la nature, il apparait indispensable d'effectuer des essais préliminaires pour connaître l'exacte toxicité des diverses substances vis-à-vis de l'insecte étudié comme de sa faune accompagnatrice. Un exemple de cette approche est fourni par l'étude de Werner et al. (1983) sur Dendroctonus rufipennis. C'est à ce prix seulement que seront utilisées les doses minimales dont les effets pervers sur la faune utile comme sur les cortèges parasitaires seront limités.

\section{- Molécules modificatrices des comportements :}

Les comportements majeurs des insectes sont régis la plupart du temps par l'émission de molécules volatiles qualifiées de composés séméiochimiques (séméion $=$ signal). Le décryptage des messages échangés par ce biais à l'intérieur d'une même espèce a ouvert la voie à diverses utilisations pratiques peu soupçonnées au départ. L'identification et la synthèse des molécules à rôle intra-spécifique (phéromones) a permis d'en découvrir les fonctions éminemment variées. Parmi elles, les phéromones sexuelles favorisant l'accouplement ouvrent d'intéressantes perspectives à l'agronome et au forestier. Encore convient-il de s'accorder sur la manière de les utiliser et sur la nature des renseignements pratiques qu'elles peuvent fournir. 
Les phéromones sexuelles possèdent souvent une structure comptant de 5 à $20 \mathrm{C}$, plus fréquemment de 7 à 13 . Ceci leur confère à la fois une spécificité suffisante en jouant sur leur configuration et un poids moléculaire assez faible (de 80 à 300) pour autoriser un haut degré de volatilité (Harbone, 1977). En dessous d'un seuil de $5 \mathrm{C}$, on tombe dans l'éventail des molécules banales dont la spécificité est limitée. Au-dessus de $15 \mathrm{C}$, les coûts énergétiques de synthèse et les problèmes de stockage deviennent plus élevés rendant ces substances plus difficiles à élaborer et à émettre (WiLson, 1972).

Par suite de leur excellente volatilité, d'infimes quantités de phéromones sexuelles exercent leur pouvoir d'attraction à de grandes distances. Les dimensions de leur espace aérien actif dépendent en partie des conditions climatiques où température de l'air, humidité, direction et force du vent jouent un rôle primordial. Dans bien des cas, la distance d'efficacité de ces molécules dépasse 100 mètres pour atteindre parfois plusieurs kilomètres. Elles offrent ainsi une méthode naturelle et inoffensive de lutte contre les populations de ravageurs.

Dans la pratique, de nombreux chercheurs rendent compte à la fois des espoirs suscités et des difficultés rencontrées dans les modalités d'application. Plusieurs approches complémentaires sont possibles :

- appâter l'insecte à l'aide de phéromones sexuelles pour déterminer sa présence et ses mouvements de populations dans le temps et l'espace ;

- déclencher des captures massives en tenant compte non seulement des possibilités techniques des pièges mais aussi des particularités biologiques des espèces ;

- essayer d'induire en confusion l'un ou l'autre des sexes par perturbation de son comportement dans une ambiance saturée.

Pour repérer la présence d'une espèce, on installe en forêt, selon un dispositif prédéterminé, des pièges naturels ou artificiels contenant des molécules actives préalablement identifiées puis synthétisées. Le contenu des pièges est alors périodiquement inventorié. Compte tenu de l'efficacité d'attraction des phéromones et de leur rayon d'influence, ce procédé permet de détecter la présence supposée d'une espèce alors que les seuils de populations sont bien trop bas pour être enregistrés par d'autres moyens. On peut ainsi dessiner la carte de la distribution d'un ravageur, en préciser l'étendue etc. C'est l'usage le plus facile des phéromones (MINKs, 1977). On ne saurait sousestimer l'avantage d'une telle détection à bas niveau de population utilisant une très faible quantité de substance. La localisation en temps utile d'une espèce nuisible permet d'appliquer diverses mesures préventives, ce qui n'était pas le cas auparavant. La prévention coûte toujours, au total, moins cher que l'éradication.

En France, divers essais utilisant des attractifs de synthèses ont eu lieu contre la Processionnaire du pin (Thaumetopoea pityocampa Schiff.) (Guerrero et al., 1981). Ces essais soulignent le rôle de divers facteurs dont on tend parfois à mésestimer l'importance (effets du type de piège, de sa position, de sa hauteur, de la dose, etc.) (Eınhorn et al., 1983). Plus généralement, il convient d'étudier divers problèmes techniques tels que le choix du substrat, des solvants, des anti-oxydants etc. (TETTE, 1974). De telles conditions d'application sont toujours à préciser. Chez les Scolytes, on doit des études analogues à divers auteurs (entre autres Vité \& PITMAN, 1970 ; BAkKE, 1973 : Nijohlt \& Schofnherr, 1977 ; Austara, 1978 ; Klimetzek, 1984 et Le Corre qui réalisa en 1984 une étude détaillée sur Ips typographus en Chartreuse).

Des essais de captures massives ont eu lieu, notamment contre les Scolytes. On doit à Bakke l'utilisation de piégeages en masse par ce procédé afin de diminuer de 
1979 à 1980 l'importance des populations d'Ips typographus sur Epicéa en Norvège du Sud et de réduire par la suite les taux d'infestation sur les arbres survivants (BAKKE, 1981 ; BAKKe \& Strand, 1981; BAKke et al., 1983). Pour ce faire, 600000 pièges furent déployés dans des parcelles réparties sur $140000 \mathrm{~km}^{2}$ (environ la surface totale de l'Allemagne de l'Est!). En 1980, 7400 Scolytes furent collectés en moyenne par piège. Au total, 2,9 milliards d'individus furent tués en 1979 et 4,5 milliards en 1980 (BAKKe, 1981). Les dommages aux arbres, dans les zones faiblement attaquées, furent réduits de façon significative autour des pièges. Ce type de piégeage, šil ne peut supprimer les épidémies après leur explosion dans les parcelles trop âgées, permet au moins de réduire les populations dans les zones moins atteintes à un niveau tel qu'elles deviennent trop clairsemées pour vaincre les réactions de défense des arbres sains. Les auteurs avouent cependant être incapables d'évaluer par ce biais l'ordre de grandeur des populations concernées. Remarquons que l'utilisation des phéromones permet d'éviter certains inconvénients liés à l'emploi d'arbres pièges qui doivent être évacués rapidement hors de la forêt peu après le début d'une attaque intense. Pour protéger les arbres avec une certaine efficacité, on peut aussi associer les techniques précédentes à l'utilisation d'insecticides (Austaka, 1978). On peut tenter d'annihiler l'un des deux sexes. Ceci a été utilisé sur divers ravageurs avec un succès plus ou moins mitigé (BIRCH, 1974). Un piégeage par annihilation est d'autant plus efficace qu'il s'adresse à des espèces géographiquement isolées. On saisit là l'intérêt des phénomènes d'insularité et la nécessité de tenir compte d'éventuels phénomènes d'immigration (BARCLAY, 1984). On peut procéder enfin par confusion en utilisant de fortes concentrations en phéromones destinées à leurrer le sexe attiré sur l'emplacement réel du partenaire recherché. Plus généralement, l'efficacité du piégeage sexuel diminue de façon importante lorsque la densité de population augmente en raison de la compétition des femelles sauvages. Par la suite, une lutte par éradication semblerait ne pouvoir être envisagée que pour daassez faibles niveaux de populations.

Un point particulier mérite attention : on tente parfois d'évaluer la densité d'une population à l'aide de phéromones. Si ce but semble être parfois atteint il convient cependant de rester prudent à cet égard. Ainsi, VoN Albert et al. (1984) ont-ils suivi l'évolution d'une population endémique d'Operophtera brumata en déterminant la relation existant entre le nombre de mâles capturés dans les pièges à phéromones et leur densité réelle dans les populations de la région. Pour ce faire, ils ont mis simultanément en ouvre pièges à glu servant de référence, pièges lumineux et plusieurs types de pièges à phéromones. La comparaison des valeurs obtenues dans ces conditions a permis de fixer l'ordre de grandeur de la densité à l'hectare de la population et l'efficacité relative des différents types de pièges. Le piégeage sexuel de Choristoneura fumiferana en Ardèche (Du Merle, 1985 a et b ; CoRnic et al., 1986) illustre les difficultés rencontrées lors de l'emploi de cette méthode. A un même nombre de captures semblent correspondre deux densités bien différentes de l'insecte. Les auteurs estiment cependant que le procédé peut être bien adapté à la surveillance des populations faibles à moyennes de ce ravageur.

Diverses molécules transmettent des informations entre individus d'espèces différentes. On les qualifie d'allomones si elles procurent un avantage adaptatif à l'individu qui les produit, par exemple en jouant un rôle défensif ou protecteur, de kairomones si elles paraissent utiles à l'organisme qui les reçoit (substances facilitant le repérage d'une proie, etc.). On peut tenter de les utiliser comme : scierie ;

- répulsifs, servant alors à protéger les arbres ou les bois stockés en forêt ou en

- attractifs vis-à-vis de parasites ou de prédateurs des ravageurs à éliminer. 
Ainsi, les larves menacées de Neodiprion sertifer relèvent l'avant du corps et régurgitent une allomone, substance visqueuse à base d' $\alpha, \beta$ pinène et d'acides résiniques extraits de la plante nourricière. Ces substances jouent un rôle dissuasif vis-àvis de l'agresseur (EISNER, 1974). Certains Hyménoptères parasites d'insectes reconnaissent leur hôte grâce à une odeur spécifique émise par celui-ci. La substance impliquée dans la reconnaissance peut provenir de la plante hôte. L'Ostomide Nemozoma elongatum (L.) détecte la présence des Scolytes Pityogenes chalcographus sur Picea abies et $P$. quadridens sur Pinus spp. grâce au Chalcogran qu'ils émettent (Heuer et VITÉ, 1984). Chez Dendroctonus pseudotsugae la M.C.H. (3-méthyl-2 cyclohexènone) est au départ une phéromone antiaggrégative naturelle (Rudinsky, 1974). Pulvérisée sur chablis, elle joue un rôle répulsif vis-à-vis des Scolytes, protégeant ainsi les troncs.

\section{- Molécules intervenant sur la physiologie de l'insecte :}

D'autres approches de lutte sont possibles basées sur l'exploitation de certaines caractéristiques physiologiques ou génétiques des Arthropodes. Le but de l'intervention humaine est alors d'interrompre le processus de développement ou de reproduction de l'insecte visé. A l'heure actuelle, deux grandes directions sont suivies par nos collègues nord américains (RETNAKARAN et al., 1982).

Les physiologistes d'insectes ont recherché des produits susceptibles de bloquer un ou plusieurs processus biochimiques fondamentaux du métabolisme. Ainsi, l'hormone juvénile (J.H.) dont on sait le rôle joué, en harmonie avec d'autres substances, dans le contrôle de la croissance et du développement de l'individu, peut-elle être utilisée pour bloquer l'insecte à sa phase larvaire l'empêchant ainsi de devenir adulte. En effet, lorsque le taux de J.H. est élevé, la mue se traduit par le passage d'un stade larvaire à un autre stade larvaire, lorsqu'il est faible la larve se nymphose chez les Holométaboles, puis elle évolue en adulte. La mise en œuvre de ce genre de molécules a suscité de grands espoirs, certains auteurs allant même jusqu'à parler d'« insecticides de

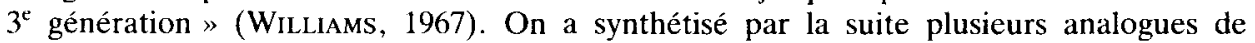
cette hormone dont l'efficacité est plusieurs fois supérieure à celle de la substance naturelle (Bowers, 1969). On en connait aujourd'hui plusieurs centaines. Certaines d'entre elles peuvent être exclusives. Ainsi, la Juvabione et le Déhydrojuvabione n'agissent que chez les Hémiptères Pyrrhocoridae.

Lorsqu'on applique un analogue de la J.H. sur des larves du dernier stade, on perturbe profondément le processus de métamorphose et l'individu mal conformé qui éclot ne survit que quelques jours. En effet, la J.H. intervient dans les processus de différenciation cellulaire, de maturation et d'embryogenèse. Elle lève aussi la diapause. On a même tenté d'appliquer cette hormone à des adultes de la Tordeuse du bourgeon de l'Epinette (Choristoneura fumiferana) ou de l'Arpenteuse de la Pruche (Lambdina fiscellaria (Hulst)) dans l'espoir de perturber le développement de la descendance. Par ce biais, la protection du feuillage de l'année n'est pas assurée et celui de l'année suivante ne l'est qu'en l'absence d'immigrations du ravageur par des individus issus des forêts voisines (RETNAKARAN et al., 1973 et 1978).

De notables succès peuvent être obtenus. Ainsi, l'usage d'analogues de la J.H. contre Coleophora aurait permis une réduction de 65 p. 100 des larves et chrysalides de la génération traitée et des cufs de la $F_{1}$, induisant ainsi une diminution marquée des dégâts causés au feuillage un an après l'application du produit (SkuHravy, 1973). Aussi l'utilisation de ce type de méthode est-elle parfois proposée plutôt chez des insectes causant des dégâts en permanence. 
On a cependant pu mettre en évidence des phénomènes de résistance aux analogues de la J.H. chez plusieurs insectes (Culex pipiens: Brown \& Brown, 1974 ; Mouche domestique : DYTE, 1962...). Là encore, il convient, pour se faire une opinion, d'attendre les résultats d'essais en vraie grandeur portant sur plusieurs générations et d'évaluer les retombées d'éventuels effets secondaires (McNeIL, 1975).

Certains dichromères inactivent la J.II. Ainsi, une inoculation d'une de ces molécules aux premiers stades larvaires produit des adultes miniatures stériles, d'où le nom de "précocène» appliqué aux produits. Une autre molécule, le fluoromévalonate, inhibe la synthèse de J.H. procurant au chercheur l'espoir de limiter l'effectif des populations d'adultes lorsque les conditions dapplications en vraie grandeur seront mieux maîtrisées (Quistadt et al., 1981). Une autre classe de composés, les benzoylurées, bloquent la synthèse de la cuticule lors du développement des insectes en empêchant la polymérisation du $\mathrm{N}$-acétyl-glucosamine, constituant élémentaire de la chitine. Les larves ayant ingéré ce produit possèdent des téguments fragiles qui se rompent lors des mues (MAAs et al., 1980). Il convient, là encore, de déterminer dans chaque cas la dose utile compte tenu des effets désirés (MADORE, 1983). Le Dimilin est classé dans cette catégorie d'inhibiteur de mue bién qu'en réalité sa structure chimique comme son mode d'action détaillé le classent à part des benzoylurées. Il interfère dans la synthèse cuticulaire en entravant le dépôt de la chitine endocuticulaire par blocage de l'incorporation de glucose sous forme de glucosamine (SALAMA et al., 1976; Quraishi, 1977). Il s'est révélé efficace contre la Processionnaire du Pin (Thaumatopoea pityocampa) (Demolin \& Millet, 1983), la Tordeuse printanière du Chêne (Croesia semipurpurana), la Nonne (Lymantria monacha). le Diprion du Pin et Boarmia bistortata (Skatula, 1975 ; Retnakaran, 1982).

Il convient de s'interroger sur l'impact de ce type de molécules sur les composants de l'écosystème autres que ceux visés. Les benzoylurées, relativement toxiques pour certains Crustacés juvéniles, seraient d'une relative inocuité pour les Oiseaux et les Mammifères, beaucoup d'Insectes et de Poissons (Buloo, 1975; Julin \& SANders, 1978; Winter, 1979). Ce type de molécules n'aurait pas d'effet mutagène ou tératogène. Ces opinions sont aujourd'hui nuancées. On sait aussi que le Diflubenzuron produit des effets toxiques sur des insectes utiles (WILKINSON et al., 1978 ; ZunGoli et al., 1983).

\subsection{Modification expérimentale du niveau nature! des populations}

On peut tenter de perturber le déroulement du cycle naturel d'une espèce lorsque celui-ci est bien connu. C'est le cas chez la Tordeuse du Mélèze Zeiraphera diniana (Baltensweiler et al., 1977). Les populations de Montgenèvre étaient en juin 1977 dans la $2^{\mathrm{e}}$ année de leur phase de régression avec une densité moyenne de 0,31 larve par $\mathrm{kg}$ de rameau. 35000 chrysalides environ ayant donné naissance à 6000 papillons furent dispersées assez uniformément dans cette forêt en juillet-août 1977. Cet accroissement artificiel du niveau des populations bloqua la phase de régression pour déclencher une accélération de la phase de progression suivie d'une moindre intensité de pullulation et de la défoliation liée à cette culmination. Durant cette progradation provoquée, diverses altérations morphologiques liées à des déficiences nutritionnelles furent constatées chez les larves ainsi qu'un taux de mortalité supérieur à la normale (Auer et al., 1981). Les perturbations du cycle gradologique pourraient être également liées à la manifestation anticipée de réactions du mélèze en relation avec l'augmentation précoce des dégâts dûs aux insectes (BENZ, 1977). 


\subsection{Procédés de lutte biologique}

On définit classiquement la lutte biologique comme l'ensemble des moyens tels que l'introduction de prédateurs, de parasitoides ou de pathogènes destinés à réguler l'évolution des populations d'insectes nuisibles. La régulation de ces populations est considérée comme réussie lorsque les dégâts sont limités à un niveau raisonnable, niveau mesuré en termes économiques ou sociologiques. Une telle approche est loin d'être récente puisque, selon Duplaquet (1970), elle fut éprouvée en forêt au Maroc dès 1925.

Comme prédateur, Calosoma sycophanta (Col. Carabidae) aiderait à réguler, dans une certaine mesure, les populations de Lymantria dispar sur chêne vert (RoL, 1955). Il consommerait $40 \%$ des nymphes de ce ravageur surtout lorsqu'elles sont localisées à la base des troncs (Weseloh, 1985 a et b). En France, il démontre une certaine efficacité contre la Processionnaire du Pin. Cependant, son faible rayon d'action autour du point de lâcher et les délais posés par l'élevage en masse d'un insecte de grande taille ne plaident pas fortement, au moins pour l'instant, en faveur de la généralisation de son utilisation à grande échelle.

On doit à GréGoIre un essai de lutte en vraie grandeur contre Dendroctonus micans dans le Massif Central par introduction aux limites de dissémination de l'espèce d'un Coléoptère prédateur Rhizophagus grandis (Grégorre et al., 1984 a et b). L'essai semble prometteur et l'on pense disposer à brève échéance d'un procédé peu onéreux de lutte contre ce Scolyte.

Des tentatives de limitation des populations de Neodiprion swainei au Québec ont été tentées par introduction à plusieurs reprises dans une forêt de Pinus banksiana de fourmis prédatrices comme Formica lugubris originaires d'Europe Centrale et $F$. obscuripes du Manitoba (Finnegan, 1974, 1975, 1977 et 1978). Diverses autres espèces de Formica ou Camponotus herculeanus joueraient un rôle analogue en régulant de façon notable les effectifs de $N$. swainei (ILNYTzky, 1974). Les résultats sont plus ambigus en ce qui concerne l'efficacité d'un Hémiptère Pentatomidae ou plusieurs Coléoptères prédateurs contre Neodiprion (TosTowarYK, 1971).

Lorsqu'on tente d'utiliser les parasitoïdes comme agents de contrôle deux stratégies sont possibles :

- le lâcher inoculatif d'un organisme qui se dissémine dans la population cible pour augmenter sa densité lorsque celle-ci est naturellement insuffisante ;

- le lâcher inondatif d'un agent de contrôle qui peut être ou ne pas être présent à l'origine dans la population.

La mise en cuvre de ces techniques n'est pas sans poser un certain nombre de problèmes. Au plan qualitatif, vaut-il mieux utiliser contre des insectes autotochnes des parasitoïdes exogènes ou locaux? Quelle est la quantité de parasitoïdes nécessaires pour réaliser un lâcher réellement inondatif par hectare de forêt lorsqu'un seuil de $1,5 \cdot 10^{5}$ ind./ha paraît minimal en agronomie? Bien des essais réalisés jusqu'à présent ont été fragmentaires et reposent sur des bases empiriques empêchant souvent de comprendre les processus fondamentaux régulant ces phénomènes (PSCHORN-WaLcher, 1977 ; Varty in Kelleher \& Hulme, 1984).

Les lâchers de parasitoïdes ont été largement utilisés au Canada aussi bien contre des insectes autochtones (C. fumiferana, Neodiprion abietis, Orgyia pseudotsugata...) qu'allochtones $(O$. brumata). Cette distinction n'est pas arbitraire. Il paraît en effet 
préférable pour obtenir un maximum de succès ccntre les espèces introduites de lutter en utilisant des parasitoïdes de même origine biogéographique qu'elles. Les lâchers réalisés par exemple au Canada contre Chorisioneura fumiferana ont produit des résultats mitigés (VARTY, op. cit.). Un Hymenoptère Eupelmidae européen, Anastatus disparis employé contre Lymantria dispar n'a pu démontrer son efficacité (Griffiths \& Quednau, in Kelleher \& Hulme, op. cit.). Des résultats plus positifs pourraient être obtenus en utilisant l'Hymenoptère Diadegma laricinellum contre Coleophora laricella (Otvos \& Quednau, in Kelleher \& Hulme, op. cit.). A l'heure actuelle, de telles approches plus ou moins empiriques, pourraient être délaissées par suite de leurs résultats aléatoires. De l'avis général, des études théoriques plus poussées sont indispensables pour mieux préciser les règles régissant l'évolution des relations entre hôte et parasitoïde.

Un hyménoptère Encyrtidae d'origine japonaise Ooencyrtus kuvanae a été introduit dans l'Ontario pour lutter contre Lymantria dispar. Il produit trois générations par an. Son impact naturel est variable, le parasitisme des cufs allant de 10 p. 100 (BRITTON, 1935 ) à 45 p. 100 (DowdEN, 1962). Si le froid prolongé tue en partie ce parasite, il semble cependant que son extension soit possible. D'autres Hymenoptères peuvent jouer un rôle analogue: par exemple l'Ichneumonide Lissonota sp., Cephaloglypta laricis et C. murinana mis en cuvre contre Choristoneura.

Les Trichogrammes parasitant les œufs de certains Lépidoptères constituent l'un des exemples illustrant le lâcher inondatif : il faut en effet en agronomie répandre $2,5 \cdot 10^{6}$ femelles de parasitoïdes à l'hectare pour avoir quelques chances de succès. En dehors des problèmes posés par la production de masse, on peut s'interroger toutefois sur l'importance de leur impact réel en forêt.

Plus généralement, la question se pose de savoir s'il faut continuer à introduire des parasitoïdes d'œufs compte tenu de leur effet réel en vraie grandeur. Au Canada, par exemple, en dehors d'O. kuvanae et de Anastatus disparis Ruschka (Hymenoptère Eupelmidae), il semble n'exister, au moins pour l'instant, aucun autre agent exploitable et même l'introduction d'un prédateur comme le Calosoma sycophanta s'y est révélée en partie inefficace contre Lymantria.

Divers parasitoïdes semblent efficaces contre la Processionnaire du Pin dont le Bombylidae Villa brunea parasite exclusif de l'espèce (Du MerLe, 1971). Son action dépend tant de facteurs climatiques que d'une certaine contexture du sol qui doit être favorable à l'enfouissement des chenilles comme à la ponte du parasitoïde. Les conditions du synchronisme spatio-temporel hôte-parasite seraient donc à favoriser. Plus généralement, l'efficacité d'un parasitoïde non spécifique pose problème : ainsi le Braconide Meteorus versicolor attaque les chenilles de Processionnaire pendant l'hiver et des ravageurs de culture à la belle saison. Une destruction inconsidérée des populations de Processionnaires détruirait un potentiel biologique important pour les cultures en éliminant l'hôte hivernal (CADAHIA et al., 1967). On touche ainsi du doigt l'une des nombreuses difficultés rencontrées dès lors qu'on effleure des problèmes de lutte biologique.

Une autre difficulté découle de l'efficacité variable d'un parasitoïde donné selon la densité de son hôte. Par exemple, l'efficacité d'Agrypon flaveolatum (Hym. Ichneumonidae) décroît lorsque la densité d'Operophtera brumata augmente. Il est par suite peu efficace aux fortes densités de population de son hôte (EMBreE, 1966). A l'inverse, Cyzenis albicans (Dipt. Tachiniidae) est bien plus efficace pour les hautes densités de la Cheimatobie (sa courbe d'accroissement devient une sigmoïde). Pour cet auteur, une 
lutte biologique raisonnée imposerait d'utiliser dans ce cas les deux espèces en fonction du niveau de population du ravageur.

Au plan méthodologique, on discute encore le fait de savoir s'il vaut mieux lâcher simultanément une ou plusieurs espèces de parasitoïdes ou de prédateurs ou s'il vaut mieux utiliser des parasitoïdes monophages ou polyphages. Le débat est donc loin d'être clos (De Bach et al., 1976 ; Clausen, 1978 ; Ehler \& Hall, 1982 et 1984...).

Une tentative d'introduction du parasite Pauesia cedrobii est en cours dans le Sud de la France pour tenter de limiter les pullulations du Puceron Cedrobium sur le Cèdre de l'Atlas. L'acclimatation semble pour l'instant réussie (FABRE \& RaBASSE, 1986).

Une autre voie d'approche consiste à pulvériser un agent pathogène vis-à-vis du ravageur à éradiquer. Plusieurs catégories d'organismes peuvent alors être employées : protozoaires, champignons, bactéries ou virus.

Plusieurs Protozoaires sont connus en agriculture pour réduire l'importance des populations de ravageurs (VARTY, 1984). Deux microsporidies, dont Nosema fumiferana ont été employées contre Choristoneura (WILSON et al., 1984). Plusieurs pulvérisations expérimentales auraient été suivies de succès. Toute application opérationnelle en vraie grandeur bute cependant sur leur production en masse.

Des Entomophthorales, comme Zoophthora radicans = Entomophthora sphaerosperma Frasenius et E. egressa MacLeod sévissent naturellement chez Choristoneura provoquant alors de 10 à 40 p. 100 de mortalité (Harvey \& Burke, 1974). Aussi des cultures de $Z$. radicans produisant conidies et spores de résistance ont-elles été entreprises sur milieu artificiel. Divers essais sont en cours destinés à améliorer l'efficacité du procédé (VANDEberg \& SoPER, 1979). En général, un climat humide favorise la dispersion et l'efficacité du champignon. Par exemple, Beauveria bassiana et plusieurs Entomophtorales sont efficaces contre Malacosoma disstria Hübner.

Parmi les bactéries, Bacillus thuringiensis en particulier a été essayé contre les Lépidoptères. Les effets pathogènes de ces bactéries reposent sur les conditions de $\mathrm{pH}$ alcalines $(9$ à 10,5) de l'intestin moyen de ces insectes. En effet, à la différence d'une bactérie formant seulement des spores, $B$. thuriengiensis secrète un cristal de protéine en plus d'endospores. Les Lépidoptères produisent en milieu basique des enzymes dissolvant la protéine cristalline et libérant de ce fait une toxine. Les larves des espèces de Lépidoptères sensibles sont alors tuées soit par la toxine soit par l'action combinée de la protéine et des endospores. De fait le procédé a été utilisé avec succès contre C. fumiferana et Malacosoma disstria Hübner. Il possède encore une certaine efficacité chez Orgyia pseudotsugata et Lymantria dispar. Il semble en revanche totalement inefficace chez Choristoneura occidentalis. Les surfaces traitées avec ce produit industriel, considéré par beaucoup comme un insecticide, peuvent être énormes $(90000$ ha au Canada). Deux traitements à grande échelle avec le $B$. thuringiensis ont été effectués dans les Alpes contre Zeiraphera diniana. Quoique leur efficacité immédiate ait été bonne $(83$ p. 100 de réduction de la population à Montgenèvre en phase de culmination avec de la Bactospeïne à $4 \mathrm{~kg} / \mathrm{ha}$ ), ils n'ont cependant pas réussi à en modifier à longue échéance le cycle de l'espèce (Grison, Martouret \& Auer, 1971 ; Martouret \& Auer, 1977). L'efficacité de ces traitements est liée à diverses pratiques qu'il convient de respecter (application du produit avant l'apparition du $4^{\mathrm{c}}$ stade larvaire du ravageur, pulvérisation du produit au niveau des cîmes, etc.). Plus généralement, il semble que le degré de succès contre une cible donnée soit fonction pour une part des techniques employées lors de la pulvérisation (au sol ou par voie aérienne, en 
phase aqueuse ou huileuse, etc.). L'une des difficultés d'application du procédé réside dans un créneau saisonnier au cours duquel on peut traiter à l'aide d'insecticides chimiques. Il existe de plus un certain délai de latence avant que des résultats soient manifestés ; ceci découle de la nécessité pour l'insecte d'ingérer la toxine alors que les insecticides chimiques procèdent pour la plupart par contact. Enfin, les préparations de Bacillus ont souvent une durée de rémanence limitée à quelques jours.

Dans le même domaine, une autre voie d'approche est constituée par l'emploi de virus. Les représentants de six familles de virus déclenchent des infections chez les insectes (voir revue d'EnTwISTLE, 1983, chez lequel on trouvera des informations détaillées).

Les baculovirus causent des polyèdroses nucléaires (V.P.N.). Ce procédé a été appliqué particulièrement contre les Neodiprion dont les larves vivent groupées. Il semble exister une dissémination naturelle du virus à partir de son point d'application. dissémination due probablement aux prédateurs et détritivores de l'espèce cible. De bons résultats ont été obtenus par exemple sur Orgya leucostigma, O. pseudotsugata, $C$. occidentalis, $L$. dispar... Des infestations expérimentales à la concentration de $10^{7}$ polyèdres par millilitre peuvent causer jusqu'à 90 F. 100 de mortalité aux $2^{\mathrm{C}}$ et $3^{\mathrm{C}}$ stades de Malacosoma; le déclenchement d'épizooties constitue l'un des facteurs fondamentaux décimant les populations (STAIRS, 1964, 1966 et 1972).

Il paraît cependant souhaitable de disposer sur le terrain des souches virales plus virulentes que les actuelles afin d'assurer un maximum de mortalité chez les ravageurs. Par ailleurs, on connait mal la persistance des virus dans le sol, ultime destination des substances; ainsi 30 à 50 p. 100 des V.P.N. d'O. pseudotsugata subsistent-ils encore dans le sol 30 ans après infestation et 1 p. 100 environ 41 ans après (THOMPSON et al., 1981).

Les éléments de réflexion sur un possible contrôle génétique ne datent pas d'aujourd'hui (DownEs, 1959 sur Lymantria dispar). Cette approche génétique a été développée assez récemment ; sa mise au point conduirait à l'ébauche de méthodes de lutte autocide avec lesquelles on espère pouvoir contrôler diverses populations. On sait que le lâcher de mâles stériles a conduit en Amérique du Nord à d'importants succès contre la Mouche des fruits. Des recherches exploratoires sont en cours sur la génétique de Choristoneura fumiferana; elles concernent la stérilité induite et diverses interférences dans la transmission des gènes. On pense pouvoir diminuer par ce biais les capacités reproductives de l'espèce lors de la phase initiale d'accroissement des populations. Bien que quelques succès paraissent avoir été obtenus sur le hanneton dans une forêt isolée (HoRBER, 1963), les perspectives d'applications opérationnelles en forêt de ces procédés ne semblent cependant pas devoir être proches (VARTY, op. cit.).

\section{Perspectives}

L'évolution des sciences contemporaines tend à indiquer que les résultats les plus prometteurs découlent souvent d'actions conduites à l'interface de deux ou plusieurs disciplines scientifiques. La science forestière ne saurait échapper à une constatation aussi banale d'où la nécessité de concevoir et de mettre en ouvre des méthodologies variées adaptées à la gamme des situations forestières et des ravageurs existants. Par suite, le fait de savoir ce qui revient au chercheur ou ce qui constitue la tâche d'un 
ingénieur de développement ne présente qu'un intérêt secondaire voire anecdotique. Si certaines approches relèvent plus particulièrement d'un métier donné, d'autres reposent sur une collaboration étroite entre ces deux catégories d'individus. L'attribution d'une tâche à l'une ou l'autre de ces catégories dépend à un moment donné des hommes, des moyens, des délais. C'est affaire de direction.

Dans une telle perspective, tout secteur de "Protection forestière " ne constitue pas seulement un organisme d'application strictement sanitaire mais aussi de recherche et de développement, approche indispensable à la mise en cuvre des méthodes et techniques nouvelles élaborées par des chercheurs plus fondamentalistes. De la collaboration entre ces deux corps dépend le succès de l'entreprise.

Une approche préliminaire à toute problématique scientifique pourrait être développée : elle concerne l'étude de l'impact réel de divers agents d'agression pour en connaître le coût économique ou sociologique. L'étude des ravageurs réellement ou potentiellement dangereux représente, dans notre pays, une charge très supérieure à celle qu'il est humainement et matériellement possible de supporter. Limportance économique des dégâts pourrait donc constituer parallèlement à l'intérêt scientifique des modèles l'un des critères de choix. Pour évaluer cet impact, la voie est ouverte: pensons aux travaux de NAIRN (1956) sur les pertes d'accroissement de Larix laricina, de Luitues (1958) sur l'impact de Diprion pini, de Cadahia \& Insua (1970), d'Oda \& Bertifilo (1980) sur Eucalyptus, de Bouchon \& Toth (1971) sur celui de la Processionnaire sur divers Pins, de JoLy (1959) sur les pertes de production du peuplier et, plus récemment, d'Archambault \& Benulieu (1985) sur les pertes d'accroissement du sapin beaumier sous l'action de Choristoneura ou de LAurent-HervoüEt (1985 a et b) sur l'impact de la Processionnaire sur Pinus pinaster et de $D$. pini sur $P$. silvestris. Une fois généralisées, de telles méthodes pourraient guider les choix surtout lorsqu'on pourra réellement chiffrer le coût financier de ces pertes, seul critère réellement pertinent pour le forestier.

Parmi les voies de recherches défrichées, certaines ont abouti. Pensons par exemple à l'utilisation à l'échelle industrielle sous diverses appellations (Bactospeïne etc.) de Bacillus thuringiensis comme insecticide. D'autres, comme l'utilisation du prédateur Rhizophagus contre le Scolyte Dendroctonus, devraient porter leurs fruits à brève échéance. Divers essais enfin nécessitent pour être conduits à bonne fin un approfondissement de nos connaissances théoriques dans des domaines scientifiques variés. Si certains de ces travaux relèvent directement de l'entomologie (problèmes d'échantillonnage, de dynamique des populations des ravageurs, de phénologie, etc.) d'autres nécessitent une étroite collaboration avec des spécialistes des disciplines voisines (génétique, physiologie animale et végétale, chimie et biochimie, etc.).

Il appartient aussi au chercheur de développer certaines approches écartées jusqu'alors. Ainsi, la synergie champignons-insectes, fort étudiée dans certains cas (liaison Ceratocystis-Scolytus de l'Orme...) a-t-elle été négligée dans d'autres. Un développement récent dans cette direction vient d'être proposé avec l'étude du rôle des champignons phytopathogènes véhiculés par les Scolytes dans les relations entre ces insectes et les conifères (LIEUTIER \& LÉvieuX, 1985). Diverses autres relations sont parfois signalées dans la littérature et par les praticiens de terrain (BonNaire, comm. pers.; Kulhavy et al., 1984) : il semble exister par exemple une certaine corrélation entre la présence de champignons comme l'Armillaire et l'intervention de Scolytes (Lessard et al., 1985). Ce type d'études conduira peut-être à analyser mieux les causes de certains dépérissements plus ou moins expliqués. Il apparaît donc indispensasble de privilégier 
une approche écologique globale des problèmes en agissant aussi bien sur l'adaptation optimale de l'essence à la station que sur le type de plantation (espacement, mixité). L'aspect protection découlera du type de gestion mis en cuvre.

Il semble bien tard pour se lancer dans l'étude des phéromones propres à certains de nos ravageurs. Il conviendrait de développer une ou deux approches de lutte biologique en explorant par exemple divers problèmes posés par la lutte génétique. Nos collègues canadiens ont depuis longtemps ouvert la voie en ce domaine et leur expérience pourrait servir de base de départ. Enfin, l'approfondissement de nos connaissances sur les mécanismes physiologiques fondamentaux des arbres, tant au plan de la phénologie du débourrement qu'à celui de la fabrication et de l'utilisation des réserves (lipides, glucides) selon les stress subis, constitue l'un des acquis à maîtriser pour tenter d'expliquer le développement de certaines populations.

Le développement de la protection forestière passe par l'entrée en ceuvre d'ingénieurs préalablement formés à cet effet. Il leur revient d'assurer en priorité formation et recyclage du personnel de terrain. Cette formation inclut la rédaction d'ouvrages pratiques illustrés facilitant sur le terrain l'identification rapide des ravageurs courants et exposant les dégâts qu'ils infligent. Simultanément, un inventaire des dégâts, présenté par exemple sur ordinateur, par forêt, région, essence, ravageurs, semble s'imposer afin de connaitre l'historique des attaques autrement que par l'anecdote et d'en retracer l'évolution.

Dans le domaine de la prévention, leur activité essentielle pourrait s'exercer dans un champ qui complète celui du chercheur. Pensons par exemple à l'étude de l'influence des facteurs sylvicoles sur le développement et la propagation initiale des populations, à la composition floristique et à la densité de la strate arbustive, au rôle de la mixité, des variations de dispositifs, de l'ornbrage relatif, etc. Ces expérimentations conduites obligatoirement en vraie grandeur nécessiteront vraisemblablement le concours de l'Office et des gestionnaires de forêts et de domaines. Il est souhaitable que les résultats, même négatifs, en soient consignés dans des documents accessibles. En effet, les forestiers ne prennent pas assez la plume pour exposer le fruit de leur expérience qui pourrait dans bien des cas guider le chercheur dans son action.

En protection, il revient en propre à ces ingénieurs d'étudier une méthodologie raisonnée d'utilisation des différents types de pièges pour en fixer les exactes modalités de mise en œuvre, connaitre par suite les seuils de population de ravageurs en période endémique, déceler leurs fluctuations avant le déclenchement de l'épidémie. Il ne suffira donc pas de s'attacher à l'importance des effectifs capturés. Enfin, diverses techniques de protection des bois peuvent être développées en précisant les coûts comparés des traitements.

Au total, les succès ne résulteront que de l'action conjointe des sylviculteurs comme celle des améliorateurs, physiologistes, pathologistes ou entomologistes unie à celle des ingénieurs de développement. L'avenir de nos forêts est à ce prix.

Reçu le 2 février 1987.

Accepté le 9 mars 1987. 


\section{Remerciements}

L'auteur tient à adresser ses vifs remerciements à Messieurs Bouvarel, Lacaze, Du Merle, Riba et Roman-Amat pour leurs critiques et suggestions. Il assure cependant l'entière responsabilité des opinions émises dans ce texte comme des erreurs ou omissions possibles.

\section{Summary \\ Pest control in forests. Present approaches and prospects}

Considering the insect infestations that occurred in some French forests for a decade, one may wonder about the efficiency of the present pest control methods and the prospects offered by current research works.

Concerning protection, a few changes in forest management methods could bring about results more rapidly than breeding of resistant varieties. Indeed, apart from noteworthy exceptions, breeding studies have to be carried out further before any possible generalization of results.

The curative methods applied so far have been more or less successful. The use of selected insecticides appeared to be very efficient against some phyllophagous insects at short term, but remained insufficient against xylophagous insects. The reasonable use of pheromone traps at low or intermediate population densities may be of some help in controlling the density of some pests. Thus, several methods are being developed, that aim at physiological modifications of the target insect while altering one of its developmental stages by means of analogues of juvenile hormones, dichromers, hormone inhibitors, benzylureas and so on.

The use of some biological control methods may be successful though involving no spectacular results. However, controlling the bark beetle Dendroctonus by Rhizophagus, a coleopterous species appears to be full of promise. Various pathogens have been tested : microsporidia, baculoviruses and so on. The efficiency of $B$. thuringiensis against several Lepidoptera has been confirmed although the remanence of preparations is often short. Studies aiming at a possible genetic control of insects have not yet been carried out further than preliminaries.

Therefore, the development of integrated control approaches and their application to forests is more than ever required, that would result from the synergistic efforts of forest specialists, geneticists and fundamental scientists.

Key words: Survey, forest, insect, review, method.

\section{Quelques ouvrages généraux}

Anderson J.F., Kaya H.K., éd., 1976. Perspectives in Forest Entomology. Acad. Press., N.Y., $428 \mathrm{p}$.

Birch M., Trammel K., Shorey H., Gaston L., Hardee D., Cameron E., Sanders C., Berard W., Wood D., Burkholder W., Muller Schwarte D., 1974. Programs utilizing pheromones in survey or control, pp. 414-462. In M.C. Birch ed., Pheromones, North Holland, Elsevier.

Coulson R.N., Witter J.A., 1984. Forest entomology. Ecology and Management. J. Wiley and Sons, N.Y., $670 \mathrm{p}$.

Dajoz R., 1980. Ecologie des Insectes Forestiers. Gauthier-Villars, Paris, 489 p.

ENTwistle P.E., 1983. Control of Insects by virus diseases. Biocontrol News and Information. C.A.B., 4, 203-229.

Harris M.K., Frederiksen R.A., 1984. Concepts and methods regarding host plant resistance to arthropods and pathogens. Ann. Rev. Phytopathol., 22, 247-272. 
Jacquiot C., 1983. Ecologie appliquée à la sylviculuure, 184 p. Gauthier-Villars ed.

Keilliher J.S., Hulme M.A., edit., 1984. Biological control of forest insect pests in Canada 19691980. In Biological Control programs against insects and weeds in Canada 1969-1980. Commonwealth Agricultural Burcau, 213-399.

Stalks G.R., 1972. Pathogenic microorganisms in the regulation of forest insect populations. Ann. Rev. Entomol., 17, 355-372.

Voute A.D., 1964. Harmonious control of forest insects, pp. 325-383. Int. Rev. For. Res., 1, J.A Romberger et P. Mikola ed., 404 p. Acad. Press., London.

\section{Références bibliographiques}

Alazaro P., 1980. Influence de la ligniculture sur les caractères de forme et de branchaison du Pin maritime. Ann. Rech. Sylvicoles A.F.O.C.E.L. 1981, 57-90.

Von Albert R., Bogensciutz A., Konic; E., 1984. Untersuchungen zum Einsatz von Sexualockstoff fallen zur überwachung des Massenswechsels von Operophtera brumata L. (Lepid. Geometridae). Z. angew. Entomol., 98, 186-198.

Archambault L., Beaulieu J., 1905. Réduction de croissance en volume occasionnée au sapin Baumier suite à la défoliation par la tordeuse des bourgeons de l'Epinette. The Forestry Chronicle, 61 (1), 10-13.

AtTard G., 1979. La tordeuse des pousses de peuplier et son cycle biologique dans le Sud-Ouest. Phytoma, 305, 23-28.

Auer C., Roques A., Goussard F, Chart.es P.J.. 1981. Effet de l'accroissement provoqué au niveau de population de la Tordeuse du Mélèze Zeiraphera diniana Guinée (Lep : Tortricidae) au cours d'une phase de régression dans un massif forestier du Briançonnais. Z. angew. Entomol., 3 (2), 286-303.

Austara O., 1978. Control of Ips typographus. Experiments with synthetic pheromones and insecticide spraying of standing trees (en norvégicn, rés. anglais). Rep. Norw. For. Inst., 34 (5), 129-152.

De Bach P., Huffaker C.B., MacPhee A.W., 1976. Evaluation of the impact of natural enemies in Huffaker C.B., Messenger P.S., pp. 225-285. Theory and practice of Biological control, Acad. Press., N.Y.

BAKKE A., 1981. The utilization of aggregation pheromone for the control of the spruce barkbeetle. A.C.S. Symposium series, $\mathrm{n}^{\circ}$ 90. Insect Pheromone technology: chemistry and it applications, 219-229.

BAKKe A., 1973. Bark beetles pheromones and their potential use in forestry. O.E.P.P. Bull., 9 , $5-15$.

Bakke A., Strand L., 1981. Pheromones and traps as part of an integrated control of the spruce bark beetle. Some results from a control program in Norway in 1979 and 1980. Rapp. nor. inst. Skogforsk. 5, 81, 1-39.

Bakke A., Saether T., Kvamme T., 1983. Mass traping of the spruce bark-beetle Ips typographus. Pheromone and trap technology. Rep. Norw. For. Inst., 38 (3), 1-35.

Baltensweller W., Benz G., Bovey P., Delucchi V., 1977. Dynamics of Larch bud moth populations. Ann. Rev. Entomol, 22, 79-100.

Barclay H.J., 1984. Pheromones trapping models for pest control : effects of mating patterns and immigration. Res. Popul. Ecol., 26, 303-311.

Beckfr M., Levy G., 1983. Le dépérissement de chêne : les causes écologiques. Exemple de la Forêt de Tronçais et premières conclusions. Rev. For. Fr., 35 (5), 341-356.

Becker M., LE TACON F., 1985. Santé de la forêt: importance d'une sylviculture adaptée aux conditions du milieu. Rev. For. Fr., no spécial, 7-28.

BELANGER R.P., 1980. Silvicultural guidelines for reducing losses to the southern pine beetle. in Thatcher R.C., J.L. Searcy, J.E. Coster and J.M. Hertel (eds). The southern pine beetle, U.S.D.A. technical bulletin 1613 .

BENZ G., 1977. Insect induced resistance as a mean of self-defense of plants. Bull. S.R.O.P., 3, 155-159. 
Bernard-Dagan C., Baradat P., 1977. Utilisation des terpènes comme outils en génétique forestière, pp. 110-132. in E.E.C. Symposium on forest trees Biochemistry, Brussels, janv. 1977.

Berthelin J., Toutain P., 1979. Biologie des sols, pp. 123-186. in Pédologie, Bonneau M. et Souchier B. (eds), tome 2, 459 p., Masson édit.

BıLoo I.J.D., 1975. Un insecticide original : le Diflubenzuron. Caractéristiques physico-chimiques. Propriétés biologiques. Mode d'action. Phytiatria-Phytopharmacie, 24 (2), 146-158.

Birch M.C., edit, 1974. Pheromones, Elsevier, North Holland.

Bouchon J., Toth J., 1971. Etudes préliminaires sur les pertes de production des pinèdes soumises aux attaques de Processionnaires du Pin Thaumetopoea pityocampa Schiff. Ann. Sci. For., 28 (3), 323-340.

Bowers W.S., 1969. Juvenile Hormone : Activity of aromatic terpenoid ethers. Science, 164, 323325.

Branham S.S., Thatcher R.C., 1985. Integrated Pest Management Research Symposium: The Proceedings, U.S.D.A. Forest Service, Gen. Techn. Report SO-56, New Orleans, Louisiana, $383 \mathrm{p}$.

BRITton G.S., 1935. The gypsy moth. Connecticut Agriculture Experimental Section Bulletin, 375 , 623-647.

Brown T.M., Brown A.W.A., 1974. Experimental induction of resistance to a juvenile hormone mimic. I. Econ. Entomol., 67, 799-801.

Cadahia D., Demolin G., Bilioti E., 1967. Meteorus versicolor Wesm. var. decoloratus Ruthe (Hym. Braconidae) parasite nouveau de Thaumatopoea pityocampa Schiff (Lep. Thaumetopoïdae). Entomophage, 12 (4), 355-361.

Cadahia D., Insua A., 1970. Estimaçion de las danos producidos por Thaumetopoea pityocampa en las repoblaciones de Pinus radiata. O.I.L.B., Coloquia de Teruel, 2-6 noviembre, 14 p.

Cameron A.E., Loerch C.R., Evans W.F., 1983. Efficacity of Sevin FR against the gypsy moth (L. Lymantriidae). J. Econ. Entomol., 76, 869-871.

Cates R.G., Alexander H., 1982. Host resistance and susceptibility, pp. 212-263. In Bark Beetles in North American Conifers, J.B. Mitton and K.B. Sturgeon edit., Univ. of Texas Press, $527 \mathrm{p}$.

Cheng H.Y., Ming W.J., Ge Q., 1983. Experiments on integrated pest control of Matsucoccus Matsumurae (en chinois). J. Nanjing techn. College of Forests Products, 1, 11-30.

Clausen C.P., edit., 1978. Introduced parasites and predators of arthropods pests and weeds : a wold review. U.S. Dept. Agric. Handb., 480.

Clerjeau M., Laterrot H., Pitrat M., 1979. Création de variétés résistantes aux maladies chez les plantes maraîchères. B.T.I. 337, 140, 101-114.

Clerjeau M., Laterrot H., Lecoo H., Pitrat M., 1981. Orientations actuelles de la sélection de variétés résistantes aux maladies chez les plantes maraîchères. Agronomie, 1 (1), 41-48.

Conntat M., 1948. Remarques sur une invasion de Lymantria dispar L. dans le cantonnement d'Uzès. Rev. For. Fr., 86 (1), 11-32.

Cornic J.F., Du Merle P., Geraud D., 1986. Piégeage sexuel de la Tordeuse du Sapin Choristoneura murinana (Lep. : Tortricidae) dans le sud de la France. Perspectives d'utilisation de cette méthode pour la surveillance des populations du ravageur. Oecol. applicata, 7 (2), 181-199.

Coulson R.N., Starck R.W., 1982. Integrated management of bark-beetles, pp. 315-349. In Barkbeetles in North American Conifers, Mitton J.B. et Sturgeon K.B. edit., Univ. of Texas Press, Austin, $527 \mathrm{p}$.

Dahlsten D.L., Dreistadt S.H., 1984. Forest Insect Pest management. Bull. Entomol. Soc. Amer., 30, 19-21.

Demolin G., Millet A., 1983. Le Dimilin utilisé à 3 doses sur le Processionnaire du Pin. Rev. For. Fr., 35 (2), 107-110.

Donley D.E., 1983. Cultural control of the red oak borer (Col. Cerambycidae) in forests management units. J. Econ. Entomol., 76 (4), 927-929.

Dowden P.B., 1962. Parasites and predators of forest insects liberated in the U.S. through 1960. U.S. Dept. Agric. Forest Service Handbook, 226, 70 p.

Downes J.A., 1959. The gypsy moth and some possibilities of the control of insects by genetical means. Canad. Entomol., 91, 661-664. 
Duplaquet L., 1970. Un essai de lutte biologique en forêt il y a près d'un demi-siècle. Rev. For. Fr., 6, 664.

Ehler L.F., Hall R.W., 1982. Evidence for competitive exclusion of introduced natural enemies in biological control. Environ. Entomol., 11, 1-4.

Ehler L.F., Hall R.W., 1984. Evidence for competitive exclusion of introduced natural enemies in biological control: an addendum. Environ. Entomol, 13 (1), V-VII.

Einhorn J., Menassieu P., Michelot D., Riom J.. 1983. Piégeage sexuel de la Processionnaire du Pin Thaumetopoea pityocampa Schiff (Lep. Notodontidae) par des attractifs de synthèse. Premiers essais dans le Sud-Ouest de la France. Agronomie, 3 (6), 499-505.

Eisner T., 1974. Depressive use by an insect of plant resin. Science, 184 (1), 996-999.

EMRREE D.G., 1966. The role of introduced parasitoids in the control of the winter moth in Nova Scotia. Can. Entomol., 102, 759-768.

Entwistle P.F., 1983. Control of insects by virus discases. Biocontrol News and Informations, C.I.B.C.. 4 (3), 203-225.

FAbre J.P., RAbasSE J.M., 1986. Introduction dans le Sud-Est de la France d'un parasite Pauesia cedrobii Stary et Leclant (Hym. Aphidiidac) du Puceron Cedrobium laportei Remaudière (Hom. Lachnidae) du Cèdre de l'Atlas: Cedrus atlantica manetti. Entomophaga, sous presse.

Fares Y., Sharpe P.A., Magnuson C.F., 1980. Pheromone dispersion in a forest ecosystem, pp. 75-93. In Stephen F.M., Scarcy J.L. et Hertel G.D. Eds " Modelling southern pine beetle populations ». Symposium proceeding. Techn. Bull. 1630, U.S.D.A. Forest Service, 174 p.

Finnegan R.J., 1974. Ants as a predator of forest pests. Entomophaga, H.S., 7, 53-59.

FinNEGAN R.J., 1975. Introduction of a predacious red wood and $F$. lugubris (Hym. Form.) from Italy to eastern Canada. Can. Entomol., 107, 1271-1274.

FinNEGAN R.J.. 1977. Establishment of a predacious red wood and F. lugubris (Hym. Form.) from Manitoba to Eastern Canada. Can. Entomol., 109, 1145-1148.

Finnegan R.J., 1978. Predation by Formica lugubris (Hym. Formicidae) on Choristoneura (Lepid. Tortricidae). Canadian Forestry Service, Bi-Monthly Research Notes, 34, 3-4.

Fraval A., Questienne P., Jarky M., 1980. Démographie de Lymantria dispar (L.) en suberaie de la Mamora : Etude le long d'un transect en 1976-1977-1978. Ann. Rech. For. Maroc, 20, $1-24$.

Geraud D., 1985. Premier bilan d'une étude de la Tordeuse du Sapin Choristoneura murinana (Lepid. Tortricidae) en Ardèche et dans les régions voisines. Aspects méthodologiques, démographiques et pratiques. Mem. E.N.I.T.E.F., $77+6 \mathrm{p}$.

Gibson K.E., BennetT D.D., 1985. Carbaryl prevents attacks on Lodgepole pine by the mountain pine beetle. $J$. of Forestry, 83, 109-112.

Giliomme J.M., Strydom D.K., Vauzy H.K., 1968. Northern Spy, Merton and Malling merton root Wocks in the Western Cape. S. af. J. agric. Sci., 11, 183-186.

Gregoire J.C., Merlin J., Pasteels J.M., Raffuel R., Vouland G., Schvester D., 1984 a. Biocontrol of Dendroctonus micans by Rhizophagus grandis in the Massif Central (France) : a first appraisal of the mass rearing and release methods. I.U.F.R.O. Working Parties 52-07-06. Göttingen, D.B.R., 13 p.

Gregoire J.C., Merlin J., Pasteels J.M., Raffuel R., Vouland G., Schvester D., 1984 b. Mass rearing and releases of Rhizophagus grandis in Lozere, pp. 122-128. In : Biological Control of bark-beetles Dendroctonus micans. Proc. Seminar E.E.C., Bruxelles, 141 p.

Grison P., Martouret D., Auer C., 1971. La lutte biologique contre la Tordeuse du Mélèze. Ann. Zool. Ecol. Anim., n" h.s., 91-121.

Guerrero A., Camps F., Coll J., Riba M., Einhorn J., Descoins C., Lallemand J.Y., 1981. Identification of a potential sex pheromone of the processionary moth, Thaumatopoea pityocampa (Lep. Notodontidae). T. Letters, 22, 2013-2016.

McGugan B.M., Coppel H.C., 1962. A review of biological control attemps against insects and weeds in Canada. II. Biological control of forest insects 1910-1958. Commonwealth Inst. of Biol. Control. Techn. Comm., 2, 35-216.

Hanover J.W., 1976. Physiology of tree resistance to insects. Ann. Rev. Entomol., 20, 75-95.

Hanover J.W., 1980. Breeding forest trees resistant to insects, pp. 487-511. In Breeding plant resistant to insects. F.G. Maxwell, P.R. Jennings ed. N.Y. J. Wiley, 683 p.

HaRborne J.B., 1977. Introduction to ecological biochemistry. Acad. Press, N.Y., 243 p. 
Harvey G.T., Burke J.M., 1974. Mortality of the spruce budworm on white spruce caused by Enteromorpha sphaerosperma. Can. Forestry Service BiMonthly Res. Note, 30, $23-24$.

Hedden R.L., Barras J.J., Coster J.E., 1980. Hazard rating systems in Forest Insect Pest management. Symp. Proc. Athens, Georgia-Gen. techn. Report U.S.D.A. Forest Service Washington D.C., $n^{\circ} \mathrm{W} 027$.

Heuer H.G., ViTE J.P., 1984. Chalcogran : unique kairomone governed predatorprey relationships among ostomid and Scolytid beetles. Naturwissenchaften, 71 (4), 214-215.

Horber E., 1963. Eradication of the white grub (Melolontha vulgaris F.) by the sterile male technique. Proc. Symp. Radiation and Radioisotopes applied to insects of Agricultural importance : I.A.E.A., 313-332.

Houston D.R., 1983. Characteristics of stands susceptible and resistant to gypsy moth defoliators, pp. 125-127. In : Proc. Forest defoliatiors-host interactions: a comparison between gypsy moth and spruce budworms, R.L. Talerico et M. Mongomery coord., Gen. Techn. Rep. Northeastern Forest and Range exp. Station, U.S.D.A. Forest-Service.

ILNYTZKY S., 1974. Formicid predators of the sawfly Neodiprion swainei Midd. Canadian Forestry Service Information Report, LAU-X-8.

Jory R., 1959. Influence des défoliateurs forestiers sur l'accroissement. Rev. For. Fr., 11, $775-784$.

Julin A.M., SANDERS H.O., 1978. Toxicity of the I.G.R., Diflubenzuron, to freshwater Invertebrates and fishes. Mosquito News, 38 (2), 257-259.

KEEP E., 1976. Breeding for resistance to Aphids in Rubus and Ribes. O.I.L.B./S.R.O.P., 3, 7983.

KLEIN W.H., 1978. Strategies and tactics for reducing losses in lodgepole pine to the mountain pine beetle by chemical and mechanical means, pp. 148-158. In A.A. Berryman, G.D. Amman, R.W. Starck and D.L. Kibbee (eds). Theory and practice of mountain pine beetle management in lodgepole pine forests. Symp. Avril 78 College of Forest Resources, Univ. of Idaho, Moscow.

KLIMETzeK D., 1984. Données de base pour une surveillance et une latte contre les Scolytidae (Trypodendron spp.) au moyen de substances attractives et répulsives (trad. en Français chez Sovilo). Freiburger Waldschutz-Abhandlungen, $207 \mathrm{p}$.

Kulhavy D.L., Partridge A.D., Stark R.W., 1984. Root diseases and blister rust associated with bark beetles (Col. Scolytidae) in western white pine in Idaho. Environ. Entomol., 13 (3), 813817.

LAurent-Hervouët N., 1986 a. Mesures des pertes de croissance radiale sur quelques espèces de Pinus dues à deux défoliateurs forestiers. I. Cas de la Processionnaire du Pin en région méditerranéenne. Ann. Sci. For., 43 (2), 71-93.

LAurent-Hervouët N., 1986 b. Mesures des pertes de croissance radiale sur quelques espèces de Pinus dues à deux défoliateurs forestiers. II. Cas du lophyre du Pin dans le Bassin Parisien. Ann. Sci. For., 43 (4), 419-440.

LE CAM J., 1983. Pinus contorta, variabilité raciale de plusieurs caractères. Ann. de Recherches sylvicoles, A.F.O.C.E.L., pp. 229-282.

Le Conre M., 1984. Utilisation de Phéromones artificielles pour l'étude de la Biologie d'Ips typographus $L$. dans le cadre d'un dispositif de piégeage après la tornade de novembre 1982. Mém. E.N.I.T.E.F., 87 p.

Lespinasse Y., Milaire H.G., Decourtye L., 1976. L'amélioration du pommier pour la résistance aux champignons parasites et aux arthropodes nuisibles. B.T.I., 306, 17-33.

Lessard G., Johnson D.W., Hinds T.E., Hoskins W.H., 1985. Association of Armillaria root disease with mountain pine beetle infestations on the Black Hills National Forest, South Dakota. U.S.D.A. Forest Service, F.P.M./M.A.G., Fort Collins, Co., Rpt 85-4.

LevieuX J., 1986. Exemples d'études de résistance génétique des arbres forestiers aux attaques d'insectes. Rev. For. Fr., n' spécial, 234-239.

Lieutier F., 1983. Les Nématodes associés aux principaux Scolytides ravageurs français : étude de la maturation d'Ips sexdentatus ; action des Nématodes parasites sur ce Scolyte. Thèse Sciences, Univ. Paris VI, $228+17 \mathrm{p}$.

Lieunier F., Levieux J., 1985. Les relations Conifères-Scolytides : importance et perspectives de recherche. Amn. Sc. For., 42 (4), 359-370.

Lindstrom A., Hellmark R., Hellquist C., 1982. Plant collars against pine weevil attacks. Design and biological evaluation. Rapport Institutionen för Skogsproduction, Sveriges Lantbruksuniversitat, $9,37 \mathrm{p}$. 
LuITJEs J., 1958. Over de economische betekenis van insektenplagen in bossen (Cephalica alpina Klüg. en Diprion pini). Dissertation 56 p., Meded. Inst. toegep. Biol. Onderr. Nat., 40.

Maas W., Hes R., Grosscourt A.C., Deul D.H., 1980. Benzoylaphenylurea Insecticides. Chemie der Pflanzenschutzund Schadlingsbekanfungsmittel, 6, 423-470.

Madore C.D., Boucias D.G., Dimond J.B., 1983. Reduction of reproduction potential in spruce budworm (L. Tortricidae) by a chitin-inhibiting insect growth regulator. J. Econ. Entomol., $76(4), 708-710$.

Martouret D., Auer C., 1977. Effet de Bacillus thuringiensis chez une population de Tordeuse grise du Mélèze Zeiraphera diniana (Lep. : Tortriciclae) en culmination gradologique. Entomophaga, 22 (1), 37-44.

Massonie G., 1977. Résultats préliminaires d'une étude sur la sensibilité du Pêcher envers le Puceron vert du Pêcher Myzus persicae Sulzer. O.I.L.B./S.R.O.P., 3, 69-73.

Mendel Z., 1984. Provenance as a factor in susceptibility of Pinus halepensis to Matsucoccus josephi. Forest Ecology and Management, 9 (4), 259-266.

Du Merle P., 1971. Sur quelques facteurs qui régissent l'efficacité de Villa brunnea Beck (Dipt. Bombylidae) dans la régulation des populations de Thaumetopoea pityocampa Schiff. (Lepid. Thaumetopoeidae), pp. 57-66. In La lutte biologique en forêt, $\mathbf{n}^{\circ}$ Hors Série, Ann. Ecol. Anim., INRA édit.

Du Merle P., 1985 a. Piégeage sexuel de Tortrix viridana L. (L. Tortricidae) en montagne méditerranéenne. 1. Epoque de vol et dispersion des adultes. Z. angew. Entomol., 100 (2), 146-163.

Du Merle P., 1985 b. Piégeage sexuel de Tortrix viridana L. en montagne méditerranéenne. 2. Relation entre le nombre de captures et le niveau de population. $Z$. angew. Entomol., 100 (3), 272-289.

MiLLER G.E., 1983. Evaluation of the effectiveness cold-water misting of trees in seed orchards for control Douglas-fir cone gall midge (Diptera Cecidomyiidae). J. Econ. Entomol., 76, 916-919.

Miller F.D., StePhen E.M., 1983. Effects of competing vegetation on Nantucket pine tip moth (Lepid. Tortricidae) populations in loblolly pine plantations in Arkansas. Environ. Entomol., $12(1), 101-105$.

MinKS A.K., 1977. Trapping with behavior modifying chemicals : feasibility and limitations, pp. 385-394. In Chemical Control of Insect behaviour, Shorey H. and McKelvey J. ed., Wiley and sons.

Mitchell R.G., Waring R.H., Pitman G.B., 1983. Thinning lodgepole pine increases tree vigor and resistance to mountain pine beetle. Forest Science, 29 (1), 204-211.

Moulson D.C., 1985. The development of a climbing tree delimber/debarker. Technical Note, Forest Engineering Research Institute of Canada, $\mathrm{n}^{\circ} \mathrm{TN} 81,17 \mathrm{p}$.

NaIRN L., 1956. Defoliation and thinning in relation to radial increment of Tamarack. Prog. Rep. Div. For. Biol., Dpt Agric., Canada, 12, 2.

Narahashi T., 1965. The Physiology of Insect Central Nervous System, J.E. Treherne and J.W.L. Beament Editor, Acad. Press, London.

McNeIL J., 1975. Juvenile Hormone analogs: Detrimental Effects on the Development of an Endoparasitoid. Science, 189, 640-642.

Nijholt W.W., Schofnilerk J., 1977. Réactions des Scolytidae aux produits chimiques en Allemagne de l'Ouest et dans l'Ouest du Canada (traduit de l'anglais). Rev. bimestrielle de Rech., $33(1), 1-12$.

OdA S., BerTIFIIo E., 1980. Incremento annual volumctrico de Eucalyptus saligna $\mathrm{Sm}$ en áreas com diferentes niveis de infestaçao de lagartas de Thyrcinteina amobia (Stoll, 1782) (Lep. Geometridae). I.P.E.F., 17, 21-3̋1.

Perrin R., 1979. Récents progrès dans les connaissances relatives à la maladie de l'écorce du Hêtre. Rev. For. Fr., 31 (3), 224-226.

Pinon J., 1984. Perspectives de la sélection pour la résistance en pathologic forestière. C.R. Acad. Agriculture France, 70, 881-888.

Pinon J., 1986. La sélection pour la résistance aux maladies. R.F.F., XXXVIII, $\mathrm{n}^{\circ}$ spécial, 228233.

Ponge J.F., 1983. Les Collemboles, indicateurs du type d'humus en milieu forestier. Résultats obtenus au sud de Paris. Oecologia generalis, 4, 35.9-374.

Ponge J.F., Vannier G., Arpin P., David J.F., 1986. Caractérisation des Humus et des litières par la faune du sol. Intérêt sylvicole, Rev. For. Fr., 38, 509-516. 
Prebble M.L., 1976. Aerial control of forest insects in Canada. Information Canada, Supply and Services, Government of Canada, Ottawa, K1A 059.

Pschorn-Walcher H., 1977. Biological control of forest insects. Ann. Rev. Entomol, $22,1-22$.

Quistad G.B., Cerf D.C., Schooley D.A., Stalls G.B., 1981. Fluoro-mevalonate acts as an inhibitor of insect juvenile hormone biosynthesis. Nature, 239, 176-177.

Quraishi M.S., 1977. Biochemical Insect Control. J. Wiley and Sons, N.Y., 280 p.

Retnakaran A., Jobin L., Buckner C.H., 1973. Experimental aerial application of a juvenile hormone analog against the Eastern Hemlock looper, Lambdina fiscellaria (Guen) in Anticosti Island in July 1973. Can. For. Serv., Inf. Rep. IP-X6.

Retnakaran A., Kaupp W., Howse G., 1978. Experimental aerial application of Insect growth regulators against the spruce budworm Choristoneura fumiferana (Clemens) in Thessalon (1976) and Hearst (1977). Can. For. Serv., Inf. Rep. FPM-X-19.

Retnakaran A., Wilson G.G., Ennis T.J., Fast P.G., Arif B.M., Tyrrel D., Wilson G.G., 1982. Développement de méthodes environnementalement acceptables pour le contrôle des ravageurs forestiers. Can. For. Serv., Inf. Rep. FPM-X-62.

Robredo F., Sanchez A., 1983. Lucha quimica contra la lagarta verde de la cucina Tortrix viridana L. (Lep. Tortricidae). Evolution de las tecnicas de applicacion desde las primeros eusayos y trabajor realizados hasta el memento actual. Bio. Servicio de Defensa contra Plagas e Inspeccion Fitopatologica, 9, 253-272.

Rodzıewicz A.. Kolk A., 1980. Effects of perennial lupins on the density of Rhyacionia buoliana and other insects in young Scots Pine plantations. Sylwan, 124 (3), 23-30.

Rol. R., 1955. La végétation forestière de la Corse. Rev. For. Fr., 2, 841-852.

Roth E.R., 1970. Resistance : a literature review of important insects and diseases. U.S. Dpt Agric. For. Service Southeastern area Rep., 28, $59 \mathrm{p}$.

Rudinsky J.A., Morgan M.E., Librey L.M., Putnam T.B., 1974. Antiaggregativerivalry Pheromone of the Mountain Pine Bectle and a new arrestant of the Southern Pine Beetle. Environ. Entomol., 3 (1), 90-98.

Russell G.E., 1978. Plant breeding for pest and disease resistance. Butterworths. London, $485 \mathrm{p}$

SAFRANYIK L., LiNTON D.A., 1982. Effect of water sprinkling of spruce logs on bark beetle attacks. Canadian Forestry Service Research Note, 2 (2), 8-9.

Salama H.S., Motagali.y Z.A., Skatulla U., 1976. On the mode of action of Dimilin on a moulting inhibitor in some Lepidopterous insects. Z. angew. Entomol., 80 (4), 396-407.

Schwerdtfeger F., 1954. Forstinsekten im Ur- und Nutzwald. Allg. Forstztg., 9, 278-282.

Schvester D., Ughetto F., 1986. Différences de sensibilité à Matsucoccus feytaudi Duc. (Homoptera : Margarodidac) selon les provenances de pin maritime (Pinus pinaster). Ann. Sci. For., 43 (4), 459-474.

Skatulla U., 1975. Über die Wirkung des Entwicklungshemmers Dimilin auf Forsinsekten. Anz. Schädlingskd. Pflanzen Unweltschutz, 48, 145-147.

Skumravy U., 1973. Field Control of the larch casebearer moth Coleophora Laricella with juvenoid. Acta entomol. Bohemoslov., 70, 313-322.

Staiks G.R., 1964. Dissemination of nuclear polyhedrosisvirus against the forest tent caterpillar Malacosoma disstria (Hübner) (Lepid. Lasiocampidae). Can. Entomol., 96, 1017-1020.

Stairs G.R., 1966. Transmission of virus in tent caterpillar populations. Can Entomol., 98, 1100 . 1104.

Steiner K., 1974. Genetic differences in resistance of Scots pine to eastern pineshoot borer. The Great Lake Entomologist, 7, 103.

Stiell W.M., Berry A.B., 1985. Limiting white pine weevil attacks by side shade. Forestrv Chronicle, 61 (1), 5-9.

Thompson C.G., Scott D.W., Wickman B.E., 1981. Long term persistance of the N.P.V. of the Douglas fir tussock moth (Orgyia pseudotsugata) (Lepid. Lymantriidae) in forest soils. Env. Entomol., 10, 254-255.

TODA R., 1974. Forest tree breeding in the world. Gov. For. Exp. Srn. Jap., Tokyo, 205 p.

Tostowaryk W., 1971. Relationship between parasitism and predation of Diprionid sawflies. Ann. Entomol. Soc. Amer., 64, 1424-1427.

Turchinskaya I.A., 1983. The silvicultural and biological basis of prophylatic measures against the Pine weevil (en russe). Lesnoe Khozyaistuo, 7, 50-51. 
VAndenberg J.D., Soper R.S., 1979. A bioassay technique for Enteromorpha sphaerosperma on the spruce budworm Choristoneura fumiferana. J. Inv. Pathol., 33, 148-154.

Van der Plank J.E., 1975. Principes of Plant Infection. Acad. Press, New-York, $216 \mathrm{p}$.

VARTY I.W., 1984. Review of biological control opportunities against spruce budworm, Choristoneura fumiferana (Clem.), pp. 273-276. In Biological Control Programs against Insects and weeds in Canada 1969-1980. J.S. Kelleher et M.A. Hulme ed. C.A.B., 410 p.

Vite J.P., Pitman G.B., 1970. Management of western pine beetle populations : use of chemical messengers. J. Econ. Entomol, 63 (4), 1132-1185.

Voute A.D., 1964. Harmonious control of forest insects, pp. 325-383. In Int. Rev. Forestry Research, vol. 1, Romberger J.A. et Mikola P. ed, Acad. Press.

Werner R.A., Hastings F.L., Averill R., 1983. Labcratory and field evaluation of insecticides against the spruce beetle (Col. Scolytidae) and parasites and predators in Canada. J. Econ. Entomol., 76, 1144-1147.

Weseloh R.M., 1985 a. Predation by Calosoma sycophanta (Col. Carabidae) : evidence for a large impact on gypsy moth : Lymantria dispar L. (Lepid. : Lymantriidae) pupae. Can. Entomol, $117(9), 1117-1126$

WeSELOH R.M., $1985 \mathrm{~b}$. Changes in population size, dispersal behavior and reproduction of Calosoma sycophanta L. (Col. : Carabidae) associated with changes in gypsy moth Lymantria dispar (Lepid. : Lymantriidae) abundance. Environ. Entomol., 14 (3), 370-377.

Wilkinson J.D., Biever K.D., Ignoffo C.M., Pons W.J., Morrison R.K., Seay R.S., 1978. Evaluation of Diflubenzuron formulations on selected insects parasitoids and predators. J. Ga. Entomol. Soc., 13, 227-236.

Williams C.M., 1967. Third generation pesticides. Sci. Am., 217, 13-17.

WIISON E.O., 1972. Chemical communication within animal species, pp. 135-136. In Chemical Ecology, E. Sondheimer et J.B. Simeone eds, Acad. Press., N.Y.

Wilson G.G., Tyrrell D., EnNIS T.J., 1984. Application of microsporidies and fungi and of genetic manipulations, pp. 260-266. In Biological Control Programm against Insects and weeds in Canada 1969-1980. J.J. Kelleher and M.A. Hulme edit., C.A.B., Farnham Royal, 410 p.

WinTER K., 1979. Untersuchungen über die Answirkungen von Dimilin auf Insekten und Spinnen der Bodenorfläche in Kieferwäldern. Mitt. Biol. Batandesanstalt für Land und Forstwirtschaft, 191, 228-229.

Witter J.A., LynCh A.M., Montcomery B.A., 1983. Managements implications of interactions between the spruce budworms and spruce-fir stands in eastern North-America, pp. 127-132. In Proc. Forest defoliators-host interactions: a comparison between gypsy moth and spruce budworms New Haven, Comm. R.L. Talerico and M. Montgomery coord. Gen. Techn. Rep., Northeastern Forest and Range exp. Station, U.S.D.A. Forest-Service.

Wright J.W., Lhmmian W.A., Bright J.N., Day M.W. Sadjak R.L., 1976. Scotch Pine varieties for Christmas trees and forest planting in Michigan. Michigan State Univ. Rescarch. Report, n" 293.

Yu C.M., Guo S.P., Cheng D.J., 1984. Study on the larch bark-bectle I.P.S. subelongatus motsch (en chinois). J. of North Eastern Forestry Institute, China, 12, 27-39.

Zungoli P.A., Steinilaver A.L., Linduska, 1983. Evaluation of Diflubenzuron for Mexican bean beetle (Col. Coccinclidac) control and impact on Pediobitus foveolatus (Hym. Eulophidae). $J$. Econ. Entomol., 76, 188-191. 